\section{Alternative Medicine Goes Mainstream}

\author{
J. Bösch
}

"Alternative Medicine Goes Mainstream", so titelte die wöchentlich in New York erscheinende Finanzzeitschrift "BARRON'S» in ihrer Ausgabe vom 15. Mai 2000 unter der Rubrik "Investing in Health». Damit wird auch dem breiteren Publikum und insbesondere den Investoren zur Kenntnis gebracht, was Forscher wie David Eisenberg der Fachwelt schon seit längerem bekannt gemacht haben: Die Konsultationen für CAM (Complementary and Alternative Medicine) hatten zwischen 1990 und 1997 eine Zuwachsrate von $50 \%$ und stiegen von 426 Millionen auf 629 Millionen. In der gleichen Zeitspanne waren die Konsultationen bei den Grundversorgern leicht rückläufig von 388 Millionen Konsultationen auf 386 Millionen. Die Bevölkerung der USA bezahlte 1997 für CAM 27 Milliarden Dollar aus eigener Tasche; dies soll ungefähr der Summe für alle ambulanten Arztkonsultationen entsprechen.

"Das erste Mal in der Geschichte der westlichen Medizin können wir eine solche von den Konsumenten bestimmte Bewegung feststellen", wird Dr. Woodson Merrell, Executive Director des "Center for Health and Healing" des "Beth Israel Hospitals» in New York zitiert. Seine Abteilung will im Jahr 2000 zwischen 35000 und 40000 Patienten mit der Kombination von CAM und konventioneller Medizin behandeln. Weltweit führende Spitäler haben die Entwicklung erkannt und entsprechend darauf reagiert. Das "Columbia Presbyterian Hospital» in New York, eines der führenden Zentren für Herzchirurgie hat auf Initiative des weltbekannten Herzklappenspezialisten Mehmet $0 z$ für 10 Millionen Dollar eine sogenannte Mind-Body-Abteilung eingerichtet, in der die Herzchirurgiepatienten nicht nur vor und nach der Operation mit Entspannungstechniken, Musiktherapie, Yoga und ähnlichem behandelt werden, sondern sogar während der Operation am offenen Herzen wird geistig-energetische Therapie (Geistheilen) appliziert. Der Erfolg und die Nachfrage veranlassten das Spital, diese Leistungen auch ambulant anzubieten und entsprechende umfassende Gesundheitsberatungen in ihr Programm aufzunehmen.

Korrespondenz:

PD Dr. med. Jakob Bösch

Kantonsspital

Externe Psychiatrische Dienste

CH-4101 Bruderholz

E-mail: jakob.boesch@kpd.ch
Inzwischen haben andere führende Spitäler wie das "Memorial Sloan-Kettering Cancer Center» oder das "Beth Israel Deaconess Medical Center» in Boston nachgezogen und mit ähnlichen Investitionen ebenfalls Mind-Body-Abteilungen eingerichtet. Diese Leistungen sind keineswegs billig, eine etwa 20minütige Konsultation kostet je nach darin enthaltenen Leistungen zwischen 75 und 350 Dollar.

Auch die medizinischen Hochschulen und die Krankenpflegeschulen sind daran, auf die Entwicklung zu reagieren. Gegenwärtig bieten ungefähr 2/3 der medizinischen Hochschulen der USA Kurse in CAM an. An einem Grossteil der Krankenpflegeschulen wird "Therapeutic Touch", eine systematisch gelehrte Form des Handauflegens bzw. der geistig-energetischen Behandlung als offizielles Fach im Lehrplan geführt. Die Entwicklung ist in diesem Bereich so stürmisch, dass die "Nurse Healers Professional Associates International Inc.» den Überblick zurzeit verloren hat.

Manches ist in den USA nicht auf Initiative von Spitälern oder Ärzten geschehen, sondern auf politischen Druck. Der amerikanische Kongress hat 1991 das "Office of Alternative Medicine» (OAM) mit einem Forschungsbudget von 2 Millionen Dollar als kleinen Zweig des "National Institute of Health" (NIH) ins Leben gerufen. Inzwischen hat sich das OAM zum NCCAM ("National Centre for Complementary and Alternative Medicine») entwickelt mit einem jährlichen Forschungsetat von 68 Millionen Dollar. Nach Stephen E. Straus, NCCAM-Direktor, geht es insbesondere darum, für Forschungsgebiete wie Bioelektromagnetische Therapie, geistig-energetische Therapie (Geistheilen), Homöopathie und Gebetsheilen eine Forschungsinfrastruktur aufzubauen, da Wissenschaftler mit solchen Themen im traditionellen Forschungsbetrieb wenig Unterstützung haben.

Es gibt auch in den USA weiterhin Feindseligkeiten gegenüber der CAM von seiten der etablierten Medizin. Dieser Widerstand gehört nicht nur in der Medizin mit zum wissenschaftlichen Fortschritt. Der Wissenschaftshistoriker Thomas Kuhn wurde Anfang der siebziger Jahre weltberühmt mit seiner Arbeit über den wissenschaftlichen Paradigmenwechsel und hat die regelmässige Dynamik solcher Wechsel anschaulich beschrieben. Schon Vesal wurde von seinen Kollegen und Lehrern bekämpft, und Newton wurde als Mystiker beschimpft, als er eine allgemeine Gravitationskraft postulierte. Und als Philipp Ignaz Semmelweiss, der die Ursache des Kindbettfiebers entdeckte bzw. eine Kontaktinfektion postulierte, seine Kollegen vor 150 Jahren aufforderte, beim Wechsel vom Autopsie- in den Gebärsaal die Kleider $\mathrm{zu}$ wechseln sowie die Instrumente und die Hände zu reinigen, sollen diese Kollegen sich einen Sport daraus gemacht haben, ihre Operationsskalpelle an den Schuhsohlen zu wetzen, um ihn zu demütigen. Seit dem Aufkommen der Naturwissenschaften ist die Geschichte voll von ähnlichen eindrücklichen Beispielen. Dass dieser Widerstand ein allgemeines Phänomen in den Wissenschaften darstellt, hat auch der Nobelpreisträger für Physik, Max Planck, dargelegt. 
Planck betonte, man solle nicht hoffen, neue Erkenntnisse seinen Kollegen beibringen zu können. Man müsse geduldig warten, bis diese ausgestorben seien, und die junge Generation wäre wie selbstverständlich mit der neuen Wahrheit vertraut. Etwas sarkastisch hat Planck seine Erfahrung in dem Satz zusammengefasst: "Die Wissenschaft verändert sich von Beerdigung zu Beerdigung". Von Sokrates ist überliefert, dass er ungefähr gesagt haben soll: "Ihr glaubt $\mathrm{zu}$ wissen, ich weiss wenigstens, dass ich nichts weiss.» Es braucht viel Grösse und viel Demut, um gerade in unserer Zeit die Kleinheit der Insel des Wissens im Meer unseres Nichtwissens zu erkennen.

Fehlende wissenschaftliche Grundlagen werden oft als Grund für den Widerstand von seiten der etablierten Medizin gegenüber CAM herangezogen. Es wird dabei übersehen, dass in manchen Fällen nicht das Fehlen wissenschaftlicher Studien, sondern die Unkenntnis dieser Arbeiten das Hauptproblem ist. So sind in sogenannten Metaanalysen (Auswertung der vorhandenen Studien) zur geistig-energetischen Behandlung und zur direkten menschlichen Beeinflussbarkeit von lebenden Systemen Signifikanzen und Effektstärken errechnet worden, wie sie bei der Untersuchung von Medikamentenwirkungen selten vorkommen. Auf der anderen Seite ist die moderne psychophysiologische und biophysikalische Forschung daran, immer mehr Puzzleteile von Erkenntnis zu liefern, so dass heute die Hypothesenbildung zur Erklärung sogenannter alternativer und feinstofflicher Therapien sehr weit gediehen ist. Beispielsweise zeigt die Biophotonenforschung, vor etwa 20 Jahren von Prof. Fritz Albert Popp in Deutschland begründet, auf, dass der menschliche Organismus über ein ähnliches, lichtschnelles Informationssystem verfügt, wie es in der modernen Informations- und Kommunikationstechnologie allgemein bekannt ist. Laut Popp gibt es heute weltweit etwa 40 Forschungsteams, die die Biophotonenforschung vorantreiben, die meisten in Japan, China und Indien. Japan soll, laut Popp, jährlich bis zu 100 Millionen Dollar in diese Forschung stecken und ein Ganzkörper-Photonen-Scanning entwickeln. Inzwischen wird die Biophotonenforschung in der Lebensmittelqualitätsprüfung auch in Europa bereits kommerzialisiert. Im medizinischen Bereich wird Europa, laut Popp, den Forschungsrückstand kaum mehr aufholen können. Interessanterweise hat die Untersuchung von Geistheilern gezeigt, dass deren Biophotonenabstrahlung während des Heilungsvorgangs bis zu 1000mal stärker sein kann gegenüber der Abstrahlung bei durchschnittlichen Menschen.

Eine andere Erkenntnisquelle für die CAM-Therapien bildet die sogenannte Biofeld- oder Auraforschung, wie sie beispielsweise die amerikanische Psychophysiologin Valery V. Hunt iniziiert hat. Hunt hat gezeigt, dass zwischen dem, was sogenannt aurasichtige Menschen wahrnehmen und dem, was elektrophysiologisch ableitbar ist, strenge Korrelationen bestehen. Durch Zusammenarbeit mit der physikalischen Abteilung an der "University of California" in Los Angeles und Benutzung deren Versuchsräume konnte Valery V. Hunt das Biofeld menschlicher Versuchspersonen manipulieren und damit zahlreiche Symptome experimentell hervorrufen, wie sie bei Epilepsie, Autismus, psychophysischem Entwicklungsrückstand und Schizophrenie vorkommen. Das gemeinsame an dieser neuen und alternativen Forschungsrichtung ist, dass sich weder Kliniker noch Forscher scheuen, Menschen in ihre Arbeit einzubeziehen, die über besondere Begabungen wie Geistheilen, Hellsichtigkeit, Aura-Sehen usw. verfügen. Ähnlich wie sich eine renommierte Musikakademie darum bemüht, besonders begabte Musiker heranzuziehen und nicht die durchschnittliche Begabung als Mass aller Dinge sieht. 